$01.1 ; 11$

\title{
Нейросетевой имитатор нестационарной среды в адаптивной системе передачи данных
}

\author{
() А.А. Невзоров, А.А. Орлов, Д.А. Станкевич \\ Волгоградский государственный университет, Волгоград, Россия \\ E-mail: terrapevt@mail.ru
}

Поступило в Редакцию 11 апреля 2019г.

В окончательной редакции 11 апреля 2019г.

Принято к публикации 12 апреля 2019г.

\begin{abstract}
Описывается новый метод оптимизации канала передачи данных, основанный на применении нейросетевого имитатора нестационарной физической среды. Эксперименты показали, что метод при малом отношении сигнал/шум позволяет уменьшить вероятность ошибки приема до уровня, соответствующего двоичной фазовой манипуляции.
\end{abstract}

Ключевые слова: нейронные сети, адаптивные системы связи, моделирование физической среды.

DOI: 10.21883/PJTF.2019.14.48013.17836

Постоянно растущий объем передаваемых данных требует разработки новых более эффективных и устойчивых к помехам способов кодирования и декодирования информации. Проблема увеличения пропускной способности каналов передачи данных особенно актуальна для нестационарных сред распространения. В последнее время на фоне роста производительности систем параллельных вычислений появилось много работ, в которых для решения указанной проблемы используются методы машинного обучения. Например, в работе [1] для компенсации искажений волнового фронта при прохождении лазерного пучка через атмосферу используется нейронная сеть, которая анализирует распределение интенсивности на приемной стороне и управляет фазовым транспарантом на передающей стороне. При этом весовые коэффициенты сети постепенно подстраиваются под изменяющиеся условия распространения оптического пучка. Использование нейронной сети позволяет за несколько сотен итераций метода обратного распространения ошибки находить распределение фазового фронта, компенсирующее искажения в турбулентной атмосфере.

В работах [2,3] предлагается заменить кодер и декодер одной нейронной сетью с архитектурой типа „автоэнекодер“. Сеть-кодер формирует из входной битовой последовательности $X$ дискретный сигнал $S_{1}$, который затем преобразуется в аналоговую форму и передается через среду распространения к приемнику, где сигнал снова дискретизируется и происходит декодирование полученной последовательности $S_{2}$ в набор битов $Y$. Обучение указанной системы заключается в таком подборе коэффициентов кодера и декодера, чтобы минимизировать число ошибок в $Y$. Для вычисления градиентов в такой сети с помощью метода обратного распространения ошибки требуется задать способ аналитического описания среды распространения. В этом случае используют достаточно упрощенные аналитические модели [4] либо в процессе обучения учитывают только аддитивную шумовую составляющую [1-3].

Авторы работы [5] предлагают тот же подход к построению кодера и декодера, что и в $[2,3]$, но при этом имитировать прохождение сигнала через среду с помощью условной генеративно-состязательной нейронной сети, состоящей из двух частей: сети-генератора и сети-дискриминатора. Генеративная сеть обучается преобразовывать сигнал с выхода кодера таким образом, чтобы сеть-дискриминатор не отличала сымитированный сигнал от прошедшего через среду. После сетьгенератор используется в качестве промежуточного слоя для вычисления градиентов ошибки в процессе обучения всей системы кодер-декодер в целом. Такой подход позволяет достаточно точно воспроизводить искажения сигнала в среде, в том числе и шумовую составляющую. Корректное обучение такой системы крайне ресурсоемкий процесс и требует использования больших обучающих множеств, что затрудняет его использование в реальных системах. Кроме того, наличие шумовой компоненты на выходе сети-генератора ухудшает прохождение градиентов ошибки при обучении кодера.

Чтобы снизить объем вычислений и иметь возможность подстраивать параметры канала связи под текущее состояние среды, подход, сформулированный в работах [2-5], предлагается модернизировать. Вместо генеративно-состязательной сети имитация среды осуществляется простой сверточной нейронной сетью, а цикл обучения разделяется на три последовательных этапа.

На первом этапе обучающего цикла декодер учится восстанавливать известную контрольную информационную последовательность $C=\left[C_{0}, C_{1}, \ldots, C_{K-1}\right]$ длиной $K$ бит по отсчетам сигнала $S_{2}$, прошедшего через реальную среду распространения. Для этого параметры сетидекодера подстраиваются так, чтобы минимизировать 
Описание архитектур нейронных сетей

\begin{tabular}{|c|c|c|c|c|}
\hline \multirow{2}{*}{$\begin{array}{c}\text { № } \\
\Pi / \Pi\end{array}$} & \multirow{2}{*}{ Характеристика } & \multicolumn{3}{|c|}{ Сеть } \\
\hline & & Кодер & Имитатор & Декодер \\
\hline \multirow[t]{3}{*}{1} & Тип слоя & Dense & Conv1D & Conv1D \\
\hline & Число нейронов & 50 & 10 & 10 \\
\hline & Активация & Leaky ReLU & Leaky ReLU & Leaky ReLU \\
\hline \multirow[t]{4}{*}{2} & Тип слоя & Conv1D & Conv1D & Conv1D \\
\hline & & & Transpose & Transpose \\
\hline & Число нейронов & 10 & 10 & 10 \\
\hline & Активация & Leaky ReLU & Leaky ReLU & Leaky ReLU \\
\hline \multirow[t]{3}{*}{3} & Тип слоя & $\begin{array}{l}\text { Conv1D } \\
\text { Transpose }\end{array}$ & Dense & Dense \\
\hline & Число нейтронов & 10 & 100 & 1 \\
\hline & Активация & Leaky ReLU & Tanh & Sigmoid \\
\hline \multirow[t]{3}{*}{4} & Тип слоя & Dense & - & - \\
\hline & Число нейронов & 100 & - & - \\
\hline & Активация & Tanh & - & - \\
\hline
\end{tabular}

перекрестную энтропию

$$
H(C, P)=-\sum_{k=0}^{K-1} C_{k} \log P_{k},
$$

$P_{k}$ - выходы сети-декодера, являющиеся оценкой вероятности того, что $k$-й бит последовательности есть логическая единица. Затем параметры сети-имитатора среды подбираются такими, чтобы минимизировать среднеквадратичное отклонение между сигналом на выходе имитатора $S_{2}^{\prime}$ и $S_{2}$. На завершающем этапе оптимизируется сеть-кодер при условии фиксированной модели среды и параметров декодера. В этом случае минимизируется перекрестная энтропия $H\left(C, P^{\prime}\right)$ исходной контрольной последовательности и оценок вероятностей приема логической единицы $P_{k}^{\prime}$ при прохождении сигнала через имитатор среды.

Исследование описанного метода обучения проводилось с использованием современных сверточных архитектур нейронных сетей, подробное описание которых приведено в таблице. Обозначения, используемые в таблице, общеприняты и описаны в [6]. В качестве оптимизатора выбран метод Adam [7], входящий в популярную библиотеку глубокого обучения Tensorflow. Время одного цикла обучения с использованием вычислительных ресурсов графического ускорителя NVIDIA GeForce GTX660 составляет приблизительно 5 ms. Кодер преобразует один бит информации в дискретную последовательность $S_{1}[n]$ длительностью $N=100$ отсчетов, причем $\left|S_{1}[n]\right| \leqslant 1$ для любого $n$. Декодер выполняет обратное преобразование получившейся после распространения через среду последовательности $S_{2}[n]$ той же длительности в один бит. Среда распространения сигнала представляет собой полосовой цифровой фильтр Баттерворта третьего порядка с частотами среза 0.1 и 0.3. После фильтрации в среде к сигналу добавляется нормальный шум с дисперсией $\sigma^{2}=64$ и нулевым средним. Таким образом, отношение сигнал/шум на приемной стороне не превышало $-4 \mathrm{~dB}$.

Через 200 циклов обучения вероятность ошибки на бит достигает 0.25 , что сравнимо с таковой для метода кодирования, использующего когерентный прием сигнала с частотной манипуляцией. Примерно после 1000 циклов вероятность ошибки приближается к предельному значению 0.2 , которое соответствует ошибке при кодировании с использованием бинарной фазовой манипуляции. Заметим, что при резком изменении параметров канала, например при сдвиге частот среза полосы пропускания на 0.1 , фазовая и частотная манипуляции перестанут работать, а нейронная сеть перестроится за несколько сотен циклов обучения.

Для описанной системы связи проводился эксперимент с простейшей моделью замирания

$$
S_{2}[n]=\left\{\begin{array}{l}
S_{1}[n]+\xi[n], n<m, \\
S_{1}[n]+A S_{1}[n-m]+\xi[n], n \geqslant m,
\end{array}\right.
$$

где $\xi[n]$ - шумовая последовательность, $A$ и $m$ выбирались для каждого сигнала случайно в диапазонах $[0.5,1]$ и $[N / 4, N / 2]$ соответственно. После резкого возникновения замирания система обучалась к новым условиям в среднем за 300 циклов, а при использовании фазовой и частотной манипуляции количество ошибок значительно увеличивалось.

Для всех описанных адаптивных систем характерно наличие дополнительного канала связи с меньшей информационной емкостью, который используется в процессе обучения. При реализации описанной системы следует учитывать, что скорость перестройки ее параметров напрямую зависит от пропускной способности дополнительного канала. Заметим также, что сетьимитатор требуется только для настройки весов кодера, поэтому выгоднее располагать ее на передающей стороне, тогда по дополнительному каналу нужно передать только оцифрованный сигнал $S_{2}$. В этом случае для вычисления $P^{\prime}$ используется копия сети-декодера на стороне передатчика, которую можно получить, повторив первый этап обучения для копии с теми же начальными значениями коэффициентов, что и у сети-декодера на приемной стороне.

Поскольку нейронная сеть является универсальным аппроксиматором, можно ожидать, что подобный подход окажется эффективным и в нелинейных нестационарных средах, характерных для систем атмосферной лазерной связи, оптоволоконных систем и в гидроакустике. При передаче информации кодер и декодер используют условия распространения, характерные для пространственного расположения излучателей и приемников, а также способ кодирования, который сложно описать алгоритмически. По всей видимости, эти факторы могут быть использованы для построения систем скрытной передачи информации. 


\section{Финансирование работы}

Исследование выполнено за счет гранта Российского научного фонда (проект № 18-79-00080).

\section{Конфликт интересов}

Авторы заявляют, что у них нет конфликта интересов.

\section{Список литературы}

[1] Lohani S., Glasser R.T. // Opt. Lett. 2018. V. 43. P. 2611-2614. DOI: $10.1364 /$ OL.43.00261

[2] Dörner S., Cammerer S., Hoydis J., Brink S. // IEEE J. Sel. Top. Sig. Proc. 2018. V. 12. P. 132-143.

DOI: $10.1109 /$ JSTSP.2017.2784180

[3] O'Shea T., Hoydis J. // IEEE Trans. Cogn. Commun. Network. 2017. V. 3. P. 563-575. DOI: 10.1109/TCCN.2017.2758370

[4] Farsad N., Goldsmith A. // IEEE Trans. Sig. Proc. 2018. V. 66. P. 5663-5678. DOI: 10.1109/TSP.2018.2868322

[5] Ye H., Li G.Y., Juang B.-H.F., Sivanesan K. // Proc. IEEE Glob. Commun. Conf. 2018. DOI: 10.1109/GLOCOMW.2018.8644250

[6] Николенко С.И., Кадурин А.А., Архангельская Е.О. Глубокое обучение. СПб.: Питер, 2018. 480 с.

[7] Kingma D., Ba J. arXiv: 1412.6980 (2014). 